\title{
VALORACIÓN DE ESTUDIOS LABORATORIALES PREQUIRÚRGICOS EN PACIENTES ASA I
}

\section{ASSESSMENT OF PRE-SURGICAL LABORATORY STUDIES IN ASA I PATIENTS}

\section{Walter DELGADO ${ }^{1}$, Fabiola MEDINA ${ }^{1}$, Christian SALA ${ }^{1}$.}

${ }^{1}$ Universidad Nacional de Asunción, Facultad de Ciencias Médicas, Departamento de Anestesiología, San Lorenzo, Paraguay.

Cómo citar este artículo: Delgado W, Medina F, Sala C. Valoración de estudios laboratoriales prequirúrgicos en pacientes ASA I. Med. clín. soc. 2019;3(2):43-49.

\section{RESUMEN}

Introducción: En los últimos años se evidenció una generalización en el pedido de pruebas preoperatorias en forma rutinaria en el preoperatorio de todos los pacientes sin que muchas veces amerite la condición clínica en vario de ellos. Objetivo: El objetivo del trabajo fue determinar la frecuencia de alteraciones en exámenes de rutina en el preoperatorio de pacientes ASA I que acudieron al Hospital Nacional de Itauguá y al Hospital de Clínicas. Metodología: Se realizó un estudio de tipo observacional descriptivo, de corte transversal en pacientes con clasificación ASA I programados para intervenciones quirúrgicas. Resultados: Se analizaron 275 pacientes en los cuales se solicitaron 11 estudios por cada paciente, lo que representó un total de 3025 estudios, de los cuales se encontraron alterados 12 estudios $(0,4 \%)$. Se constató hemoglobina alterada en un solo paciente $(0,36 \%)$, crasis sanguínea alterada en 4 pacientes (1,5\%), electrolitos alterados en 4 pacientes $(1,5 \%)$ y alteraciones en el electrocardiograma en 3 pacientes (1\%). Un escaso número de estudios solicitados se encontraron alterados. Discusión: No se encontraron alteraciones en los resultados de rutina prequirúrgicos de glóbulos blancos, plaquetas, urea, glicemia, creatinina y orina simple. Ninguna de las alteraciones modificó la técnica anestésica prevista.

Palabras clave: Exámenes preoperatorios; intervención quirúrgica; periodo perioperatorio.

\section{ABSTRACT}

Introduction: In recent years there has been a generalization in the request for preoperative tests routinely in the preoperative period of all patients without many times meriting the clinical condition in several of them. Objective: The objective of the work was to determine the frequency of alterations in routine examinations in the preoperative period of ASA I patients who attended the National Hospital of Itauguá and the Hospital de Clínicas. Methodology: A descriptive, cross-sectional observational study was conducted in patients with ASA I classification scheduled for surgical interventions. Results: 275 patients were analyzed in which 11 studies were requested for each patient, which represented a total of 3025 studies, of which 12 studies were found altered $(0.4 \%)$. Altered hemoglobin was found in a single patient $(0.36 \%)$, altered blood crasis in 4 patients $(1.5 \%)$, altered electrolytes in 4 patients (1.5\%) and electrocardiogram abnormalities in 3 patients (1\%). A small number of requested studies were altered. Discussion: No alterations were found in the routine presurgical results of white blood cells, platelets, urea, glycemia, creatinine and simple urine. None of the alterations modified the planned anesthetic technique.

Keywords: preoperative exams; surgical intervention; perioperative period 


\section{INTRODUCCIÓN}

Con mucha frecuencia se ven en el consultorio prequirúrgico de anestesiología a pacientes sanos quienes presentan numerosos exámenes de laboratorio y de imágenes antes de someterse a cualquier procedimiento quirúrgico. Estos exámenes muchas veces son innecesarios considerando la condición asintomática, sin ningún signo o antecedentes clínicos del paciente que justifique el pedido de dichos exámenes.

En los últimos años se ha evidenciado una generalización en el pedido de pruebas preoperatorias en forma rutinaria en todos los pacientes del Hospital Nacional de Itauguá y en el Hospital de Clínicas sin que amerite la condición clínica en algunos de ellos. La necesidad de racionalizar la prescripción de los exámenes complementarios se va imponiendo de manera progresiva (1-4).

Numerosos estudios han demostrado que los exámenes sistemáticos o rutinarios practicados en pacientes asintomáticos rara vez detectan anomalías que tengan un impacto sobre el periodo preoperatorio. Estos exámenes no alcanzan los dos objetivos principales en estos pacientes: la modificación eventual de la técnica anestésica y la previsión de las complicaciones postoperatorias (5).

El pedido rutinario de los exámenes preoperatorios muchas veces se ve influenciado por el temor existente a demanda judicial y dejando de lado el criterio clínico. La revisión reciente de la literatura científica indica que las pruebas preoperatorias alcanzan los mayores beneficios para los pacientes y para la sociedad si se guían por el conocimiento científico (6).

Muchas investigaciones suponen que los médicos olvidan la historia clínica y solo utilizan el laboratorio para buscar un diagnóstico. Sin embargo, la literatura demuestra fuertemente que una buena historia clínica y el examen físico en la mayoría de los casos es más efectivo. La combinación de una historia clínica acompañada de exámenes laboratoriales justificados reducen y evitan la realización de muchos exámenes laboratoriales o de imágenes innecesarios (7).

Una adecuada valoración clínica preoperatoria de los pacientes es más importante que los tests preoperatorios de rutina, que solo deberían ser pedidos cuando exista una condición clínica que lo justifique (8). El escaso beneficio que supone la realización de estudios preoperatorios rutinarios a las personas asintomáticas programadas para cirugía, junto con los altos costos, pérdida de tiempo e incomodidades para los pacientes durante la realización de las pruebas, nos obliga a reconsiderar las rutinas habituales y a promover prácticas preoperatorias basadas en la evidencia de efectividad (6).

Resulta importante evaluar la utilidad de los estudios preoperatorios en la cirugía electiva, planificada en pacientes jóvenes sin patología previa concomitante, diferente a la que motiva su consulta (9). El objetivo del estudio fue determinar la frecuencia de alteraciones detectadas en exámenes de rutina en el preoperatorio de pacientes sanos ASA I que acuden al Hospital Nacional de Itauguá y Hospital de Clínicas. 


\section{METODOLOGÍA}

Se realizó un estudio de tipo observacional descriptivo, de corte transversal en pacientes con clasificación ASA I programados para intervenciones quirúrgicas en el Hospital Nacional de Itauguá y Hospital de Clínicas durante el periodo comprendido entre enero y noviembre de 2015.

Se incluyó a pacientes con clasificación ASA I, de ambos sexos, mayores de 15 años, programados para cirugías electivas y que presentaron todos los estudios preoperatorios de rutina requeridos en el Hospital Nacional de Itauguá y en el Hospital de Clínicas.

El muestreo fue no probabilístico de casos consecutivos. Para la recolección de la información se elaboró un formulario de acuerdo a los objetivos planteados tomando como instrumento la operacionalización de las variables.

Se evaluaron si los estudios preoperatorios de los pacientes incluidos en el estudio se encontraban o no dentro de los límites normales.

Las variables involucradas fueron: sexo (masculino o femenino), edad (en años cumplidos), especialidad quirúrgica (otorrinolaringología, cirugía general, cirugía vascular, traumatología, cirugía maxilofacial, mastología, urología, oftalmología, neurocirugía).

Las siguientes variables fueron clasificadas en forma dicotómica como normal o anormal:

Electrocardiograma: normal (ritmo regular, frecuencia cardiaca de 60-100 por minuto, sin prolongación del intervalo PR ni variaciones del segmento ST).

- Hemoglobina: normal (12-14 g/dl en mujeres y $14-16 \mathrm{~g} / \mathrm{dl}$ en varones).

- Hematocrito: normal (36-44 \% en mujeres y 40-50 \% en varones).

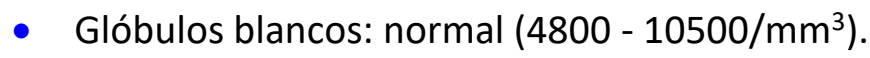

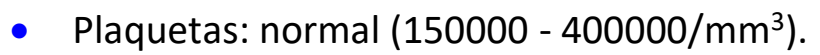

- Crasis: TP normal > $60 \%$.

- Electrolitos: normales (Na: 136-146 mEq/l; K: 3,5-5 mEq/l; Cl: 102-109 mMol/l; Ca: 8,710,2 mg/dl; Mg: 1,5-2,3 mg/dl).

- Urea: normal (7-20 mg/dl).

- Creatinina: normal (0,7 a 1,3 mg/dl en hombres y 0,5-1,2 mg/dl en mujeres).

- Glicemia: normal (70-105 mg/dl).

- Radiografía de tórax: normal (índice cardiotorácico conservado, senos costofrénicos libres, sin imágenes agregadas).

Los datos obtenidos fueron plasmados en una planilla Excel y analizados con estadística descriptiva.

\section{RESULTADOS}

Se incluyeron a un total de 275 pacientes que tenían indicación de cirugía programada. Se solicitaron 11 estudios por cada paciente, lo que representó un total de 3025 estudios. Del total, se encontraron alterados 12 estudios $(0,4 \%)$. 
Los pacientes seleccionados tenían una edad promedio de $33,97 \pm 14,4$ años, con una edad máxima de 75 años y mínima de 15 años. En cuanto a la distribución según el género, el 46\% (126 pacientes) fueron del sexo femenino y el 54\% (149) del sexo masculino. Analizando los grupos etarios se constató un $83 \%$ de pacientes adultos jóvenes, un $13 \%$ de adolescentes y un $4 \%$ de adultos mayores.

De las cirugías programadas, el 33\% fue de tipo traumatológica, 30\% cirugía general, 20\% urológica, $8 \%$ otorrinolaringológica, $3 \%$ maxilofacial, $2 \%$ vascular, $2 \%$ cirugía de mamas, $1 \%$ oftalmológica y $1 \%$ neurocirugía.

En cuanto a los hallazgos laboratoriales se constató hemoglobina alterada en 1 paciente $(0,36 \%)$. No se encontraron resultados alterados en los estudios preoperatorios concernientes a recuento de glóbulos blancos, hematocrito, plaquetas, urea, glicemia, creatinina, orina simple y radiografía de tórax.

Con respecto a los resultados de electrolitos, 4 pacientes $(1,5 \%)$ presentaron resultados alterados. En cuanto a los métodos de imágenes se encontró alteraciones en el electrocardiograma en 3 pacientes (1\%). Con respecto a la crasis sanguínea, la misma se encontró alterada en 4 pacientes $(1,5 \%)$. En la tabla 1 se hace un resumen de la frecuencia de resultados alterados que se encuentran en los estudios preoperatorios.

TABLA 1. FRECUENCIA DE ALTERACIÓN DE ESTUDIOS PREOPERATORIOS DE RUTINA SOLICITADOS EN PACIENTES. VALORACIÓN DE ESTUDIOS PREOPERATORIOS EN PACIENTES ASA I. HOSPITAL NACIONAL DE ITAUGUÁ - HOSPITAL DE CLÍNICAS. 2015 ( $\mathrm{N=275)}$

\begin{tabular}{|l|c|c|}
\hline \multicolumn{1}{|c|}{ Tipo de estudio } & $\begin{array}{c}\text { Frecuencia de resultados norma- } \\
\text { les }\end{array}$ & $\begin{array}{c}\text { Frecuencia de resultados altera- } \\
\text { dos }\end{array}$ \\
\hline Hemoglobina & $98,5 \%$ & $0,36 \%$ \\
\hline Hematocrito & $100 \%$ & $0 \%$ \\
\hline Crasis sanguínea & $98,5 \%$ & $1,5 \%$ \\
\hline Glóbulos blancos & $100 \%$ & $0 \%$ \\
\hline Urea & $100 \%$ & $0 \%$ \\
\hline Creatinina & $100 \%$ & $0 \%$ \\
\hline Plaquetas & $100 \%$ & $0 \%$ \\
\hline Orina simple & $100 \%$ & $0 \%$ \\
\hline Electrolitos & $98,5 \%$ & $1,5 \%$ \\
\hline ECG & $99 \%$ & $1 \%$ \\
\hline Radiografía de tórax & $100 \%$ & $0 \%$ \\
\hline
\end{tabular}

\section{DISCUSIÓN}

Analizando todos los estudios preoperatorios que se solicitaron en los pacientes ASA I se encontró una frecuencia muy baja de resultados alterados $(0,4 \%)$, y además en ningún caso la alteración produjo alguna modificación en la técnica anestésica propuesta.

Se realizaron más de 3000 estudios en pacientes sanos, esto representa un gasto muy importante para el hospital y por ende para la salud pública. Además, genera ocupación de turnos que podrían ser aprovechados por pacientes que están enfermos y necesitan con mayor celeridad de estudios auxiliares para hacer un diagnóstico de la patología o modificar el tratamiento. 
Pero el miedo que genera la posibilidad de incurrir en algún error que posteriormente produzca una querella en el ámbito médico-legal, es lo que hace que el médico se "cubra las espaldas" solicitando una gran cantidad de estudios que no son necesarios.

En el año 1976, Olsen y cols. concluyeron en un estudio con más de 1500 pacientes que fueron sometidos a exámenes preoperatorios de rutina, que no existen diferencias en la morbimortalidad perioperatoria si estos son solicitados o no (10). En el mismo año, Durbridge y cols. demostraron, utilizando más de 8000 exámenes preoperatorios realizados en la admisión del hospital, que no hay beneficios en la morbimortalidad del paciente ni en la estadía hospitalaria si estos son realizados rutinariamente (11).

En el año 1991, Narr y cols. demostraron que no hay cambios en la morbimortalidad perioperatoria en pacientes con clasificación ASA I al omitir los "exámenes de rutina", por lo que desde ese año en la Clínica Mayo ya no se solicitan exámenes preoperatorios rutinarios en pacientes ASA I, disminuyendo así los costos y mejorando la eficiencia del sistema (12).

En el año 1997, Munro y cols. también señalaron que no existe evidencia que apoye el uso de exámenes preoperatorios llamados de rutina (13). En el año 2012, el National Institute of Clinical Excellence (14) realizó una larga recomendación sobre los exámenes preoperatorios necesarios para cirugías electivas, la cual es de tediosa lectura y difícil aplicación en la práctica quirúrgica. Un estudio realizado por De Sousa y cols. (15) en el año 2013, concluyó que en los pacientes en los cuales se realizó un ECG de rutina se cambió la conducta perioperatoria en menos del $1 \%$ de los pacientes.

En la actualidad no existe ninguna guía o recomendación que avale el uso rutinario de hemoglobina/hematocrito ( $\mathrm{hb} / \mathrm{hto}$ ) como examen preoperatorio. En primer lugar, en pacientes sometidos a cirugía de bajo riesgo quirúrgico, menos del 1,5\% de los pacientes tenía alteraciones en el control de hb/hto y solo un $0,1 \%$ tenía alguna alteración que podría haber cambiado la conducta pre o intraoperatoria. Por otro lado, en pacientes de bajo riesgo quirúrgico el uso rutinario de hb/hto no cambió el plan anestésico o detectó nuevas patologías. La solicitud de $\mathrm{hb} / \mathrm{hto}$ debe realizarse cuando exista historia sugerente o síntomas de anemia crónica, patología de base que pudiera producir sangrado y/o anemia crónicos, y riesgo quirúrgico importante de hemorragia mayor intra o postoperatoria, en los cuales es vital tener un control de hb/hto basal (16).

Johansson y cols. (17) realizaron un metaanálisis de 23 estudios relativos al tema, encontrando correlación positiva, estadísticamente significativa entre alteraciones de la función renal preoperatorias y aumento de la morbimortalidad perioperatoria en 11 de ellos. Sin embargo, los 11 estudios mencionados incluían pacientes con enfermedades renales preexistentes, lo que quita significancia a los hallazgos encontrados. De Sousa y cols (15) estudiaron la función renal con exámenes preoperatorios de rutina (nitrógeno ureico y creatinina plasmática) en pacientes sanos sometidos a cirugía electiva de bajo riesgo, no obteniendo ningún resultado anormal, por lo que no hubo ningún cambio de conducta quirúrgica ni anestésica en el periodo perioperatorio. Una guía recomendó el uso rutinario de creatinina plasmática en pacientes mayores de 40 años sometidos a cirugía electiva (14).

Desde el punto de vista estadístico, ya en la década de los 80, investigadores como Kaplan y cols., Turnbull y Buck (18) entre otros, plantearon análisis retrospectivos de la utilización de 
los exámenes de rutina, ambos con resultados muy similares, donde se demuestra que hasta un $60 \%$ de estos fueron innecesarios y que los resultados serían previstos con una buena historia clínica y examen físico.

Por otra parte, estudios como los de Billings y Roizens mostraron que del $30 \%$ al $95 \%$ de los resultados inesperados obtenidos en las pruebas de rutina no fueron consignados en el expediente o no fueron analizados con detalle, derivando en un hecho importante: la responsabilidad médico-legal (19).

A pesar de ser tan común, hay escasa evidencia de la necesidad de los exámenes de laboratorios perioperatorios. Sin embargo, el común denominador es que éstos aportan muy poco e, incluso, pueden perjudicar al paciente, médico o institución, pues su utilidad para predecir morbilidad o modificar conductas anestésicas es muy deficiente (20).

Finalmente, una buena historia clínica junto con un examen físico completo es lo que determinará la probabilidad de un hallazgo alterado laboratorial o imagenológicamente. Racionalizar los pedidos de estudios en pacientes ASA I generará un ahorro importante al centro quirúrgico en tiempo y dinero, por lo que debe ser un tema a evaluar entre los médicos y directivos.

En conclusión, solo el $0,4 \%$ de todos los estudios solicitados a los pacientes ASA I se encontraron alterados. Se hallaron alteraciones en el dosaje de hemoglobina, electrolitos, electrocardiograma y crasis sanguínea. No se encontraron alteraciones en los resultados de rutina prequirúrgicos de glóbulos blancos, plaquetas, urea, creatinina, glicemia y orina simple. Ninguna de las alteraciones modificó la técnica anestésica prevista.

\section{CONFLICTOS DE INTERÉS Y FUENTE DE FINANCIACIÓN}

Los autores declaran no poseer conflictos de interés. Fuente de financiación: ninguna.

\section{REFERENCIAS BIBLIOGRÁFICAS}

1. Johnson RK, Mortimer AJ. Routine pre-operative blood testing: is it necessary? Anaesthesia 2002;57:914-7. https://doi.org/10.1046/j.1365-2044.2002.02750.x

2. García-Miguel FJ, García Caballero J, Gómez de Caso-Canto JA. Indications for electrocardiogram in the pre-operative asessment for programmed surgery. Rev Esp Anestesiol Reanim 2002;49:5-12. URL.

3. García-Miguel FJ, García Caballero J, Gómez de Caso-Canto JA. Indicaciones de la radiografía de tórax para la valoración preoperatoria en cirugía programada. Rev Esp Anestesiol Reanim 2002;49:80-8. URL.

4. Callaghan LC, Edwards ND, Reilly NC. Utilization of the pre-operative ECG. Anaesthesia. 1995;50:488-490. https://doi.org/10.1111/j.1365-2044.1995.tb06035.x

5. Haberer JP. Consulta preanestésica. In: Enciclopédie Médico Chirurgicale. Editions Scientifiques et Medicales Elsevier. Paris. 2001;36-375-A-05 pp 6

6. López-Bastida J, Serrano P, Duque-González B. et al. Análisis de costes y ahorros potenciales relacionados con la utilización de pruebas preoperatorias en los hospitales de Canarias. Gac Sanit 2003;17 (2):131-136. URL.

7. Roizen M. Pre-operative evaluation. In: Miller's Anesthesia. 6th ed. Elsevier. Philadelfia. 2006. Pp 927-994 
8. García FJ, Serrano PG, López-Bastida J. Pre-operative asessment. Lancet. 2003;362(9397):1749-57. https://doi.org/10.1016/s0140-6736(03)14857-x

9. American Society of Anesthesiologists. Practice Advisory for pre-anestesia evauation: A report by the American Society of Anesthesiologist task forcé on pre-anestesia evaluation. Anesthesiology 2002;96(2):485-96. https://doi.org/10.1097/00000542200202000-00037

10. Olsen D, Kane R, Proctor P. A controlled trial of multiphasic screening. N Engl J Med. 1976;294:925. https://doi.org/10.1056/NEJM197604222941705

11. Durbridge T, Edwards F, Edwards R, Atkinson M. Evaluation of benefits of screening tests done inmediately on admission to hospital. Clin Chem. 1976;22:968-71. URL.

12. Narr B, Hansen T, Warner M. Preoperative laboratory screening in healthy Mayo patients: Cost-effective elimination of tests and unchanged outcomes. Mayo Clin Proc. 1991;66:155-9. https://doi.org/10.1016/s0025-6196(12)60487-x

13. Munro J, Booth A, Nicholl J. Routine pre-operative testing: a systematic review of the evidence. Health Technol Assess 1997;1:1-62. URL.

14. Romano $P$, Schembri $M$, Rainwater J. Can administrative data be used to ascertain clinically significant pos-operative complication?. Am J Med Qual 2002; 17(4): 145-55. https://doi.org/10.1177/106286060201700404

15. De Sousa D, Marques R, Nogueira M, Fernandes V, Vieira A, Santana E. Relevancia de los exámenes de rutina en pacientes de bajo riesgo sometidos a cirugías menores. Rev Bras Anestesiol. 2013;63:197-201. https://doi.org/10.1016/j.bjanes.2012.04.004

16. Institute for Clinical Systems Improvement. Health care guideline: pre-operative evaluation. 10th ed. Bloomington, Minn: Institute for Clinical Systems Improvement; 2012

17. Johansson T, Fritsch G, Flamm M, Hansbauer B, Bachofner N, Mann E, et al. Effectiveness of non-cardiac pre-operative testing in non-cardiac elective surgery: a systematic review. Br J Anaesth. 2013;110:926-39. https://doi.org/10.1093/bja/aet071

18. Turnbull JM, Buvk C: The value of pre-operative screening investigations in otherwise healthy individuals. Arch Intern Med,1987;147:1101. URL.

19. Billings PJ, Richards R, Davies JP, Audrey DA. An audit of pre-operative investigation of surgical patients. Ann R Coll Surg Engl 75:205-93. URL.

20. Ibarra Pedro. ¿Cuáles exámenes de laboratorio preanestésicos se necesitan en pacientes asintomáticos?. Rev colomb anestesiol. 2007;35(4):301-312. URL. 